\title{
GWAS identifies a wheat orthologue of the rice D11 gene as an important contributor to grain size in an international collection of hexaploid wheat
}

Honoré Tekeu

Université Laval

Eddy L.M. Ngonkeu

Institute of Agricultural Research for Development

Sébastien Bélanger

University of Missouri

Pierre F. Djocgoué

Université de Yaoundé I

Amina Abed

Université Laval

Davoud Torkamaneh

University of Guelph

Brian Boyle

Université Laval

Patrick Tsimi

Université de Yaoundé I

Wuletaw Tadesse

International Center for Agricultural Research in the Dry Areas

Martine Jean

Université Laval

François Belzile ( $\nabla$ francois.belzile@fsaa.ulaval.ca )

Université Laval

\section{Research Article}

Keywords: rice, wheat, productivity, grain size

Posted Date: March 2nd, 2021

DOI: https://doi.org/10.21203/rs.3.rs-244194/v1 
License: (c) (i) This work is licensed under a Creative Commons Attribution 4.0 International License. Read Full License 


\section{Abstract}

Grain size is a key agronomic trait that contributes to grain yield in hexaploid wheat. Grain length and width were evaluated in an international collection of 159 wheat accessions. These accessions were genetically characterized using a genotyping-by-sequencing (GBS) protocol that produced 73,784 single nucleotide polymorphism (SNP) markers. GBS-derived genotype calls obtained on Chinese Spring proved extremely accurate when compared to the reference (>99.9\%) and showed $>95 \%$ agreement with calls made at SNP loci shared with the 90K SNP array on a subset of 71 Canadian wheat accessions for which both types of data were available. This indicates that GBS can yield a large amount of highly accurate SNP data in hexaploid wheat. The genetic diversity analysis performed using this set of SNP markers revealed the presence of six distinct groups within this collection. A GWAS was conducted to uncover genomic regions controlling variation for grain length and width. In total, seven SNPs were found to be associated with one or both traits, identifying three quantitative trait loci (QTLs) located on chromosomes 1D, 2D and 4A. In the vicinity of the peak SNP on chromosome 2D, we found a promising candidate gene (TraesCS2D01G331100), whose rice ortholog (D11) had previously been reported to be involved in the regulation of grain size. These markers will be useful in breeding for enhanced wheat productivity.

\section{Introduction}

The grain size, which is associated with yield and milling quality, is one of the essential traits that have been subject to selection during domestication and breeding in hexaploid wheat ${ }^{1}$. During the domestication process from ancestral (Einkorn) to common wheat (Triticum aestivum L.) going through tetraploid species, wheat abruptly changed, from a grain with greater variability in size and shape to grain with higher width and lower length ${ }^{2,3}$. However, grain yield is determined by two components namely, the number of grains per square meter and grain weight. Following, grain weight is estimated by grain length, width, and area, which are components showing higher heritability than mainly yield in wheat ${ }^{4}$.

Larger grains may have a positive effect on seedling vigor and contribute to increased yield ${ }^{5}$. Geometric models have indicated that changes in grain size and shape could result in increases in flour yield of up to $5 \%{ }^{6}$. Consequently, quantitative trait loci (QTLs) or genes governing grain shape and size are of interest for domestication and breeding purposes ${ }^{7,8}$. Many genetic mapping studies have reported QTLs for grain size and shape in wheat cultivars ${ }^{1,2,8-10}$ and some studies have revealed that the $D$ genome of common wheat, derived from Aegilops tauschii, contains important traits of interest for wheat breeding $^{11,12}$.

At the genomic level, Okamoto et al. ${ }^{13}$ performed QTL analyses for grain size and shape-related traits using four synthetic wheat F2 populations to identify the genetic loci responsible for grain size and shape variation in hexaploid wheat and found QTLs for grain length and width on chromosomes 1D and 2D. This is particularly interesting as the tenacious glume gene Tg-D1 on chromosome 2D is a well-known locus that has been recruited for the domestication of wheat grain size and shape. During allohexaploid 
wheat speciation, a dramatic change in grain shape occurred due to a mutation in the Tg-D1 gene $\mathrm{g}^{14}$. Furthermore, Yan et al. ${ }^{15}$ reported a genomic region associated with grain size on chromosome 2D.

New advances in genomics technologies has revolutionized research in plants by developing new high throughput genotyping methods to increase knowledge of the genetic basis of diversity in large core collection of genetic materials through genome-wide association studies (GWAS). Based on such highdensity SNP markers, GWAS can be used for the description and high-resolution mapping of genetic variance from collections of genetic ressources that have derived from several historical recombination cycles ${ }^{16}$. Furthermore, Genotyping-by-sequencing (GBS) is a Next-Generation Sequencing (NGS) technology for high-throughput and cost-effective genotyping, that provides a great potential for applying GWAS to reveal the genetic bases of agronomic traits in wheat ${ }^{17}$. Arora et al. ${ }^{18}$ conducted GWAS in a collection of Ae. tauschii accessions for grain traits, using SNP markers based on GBS. They identified a total of 17 SNPs associated with granulometric characteristics distributed over all seven chromosomes, with chromosomes 2D, 5D, and 6D harboring the most important marker-trait associations. On the other hand, most studies on germplasm of hexaploid wheat have focused on understanding the genetic and morphological diversity of this species. No studies have used GWAS based on GBS for economically important and essential grain yield components traits such as grain length and width in an international collection of hexaploid wheat. The present investigation aimed to identify QTLs and candidate genes governing grain length and width in an international collection of hexaploid wheat using a GBS-GWAS approach.

\section{Results}

Phenotypic characterization of grain yield components. To explore components of grain yield in wheat, we measured four phenotypes: grain length (Gle), grain width (Gwi), 1000-grain weight (Gwe) and grain yield (Gyi) over two years at two sites. As shown in Table 1, means ( \pm standard deviation) observed for these traits corresponded to: $3.2 \mathrm{~mm}( \pm 0.08)$ for grain length, $1.6 \mathrm{~mm}( \pm 0.04)$ for grain width, $25.7 \mathrm{~g} \mathrm{(} \pm$ $0.80)$ for 1000 -grain weight and $2.6 \mathrm{t} / \mathrm{ha}( \pm 0.11)$ for grain yield. The broad-sense heritability estimates were $90.6 \%$ for grain length, $97.9 \%$ for grain width, $61.6 \%$ for 1000 -grain weight and $56.0 \%$ for grain yield. An analysis of variance revealed significant differences due to genotypes $(G)$ for all traits and, for two traits (Gwe and Gyi), the interaction between genotype and environment (GxE) proved significant. A correlation analysis showed a high significant positive correlation between grain yield and grain weight $(r$ $=0.96 ; p<0.01)$ and also between grain length and grain width $(r=0.88 ; p<0.01)$. Also, significant positive correlations were identified between grain yield and grain length $(r=0.51 ; p<0.01)$ and between grain yield and grain width $(r=0.54 ; p<0.01)$. Interestingly, a bimodal distribution was observed for grain length and width (Fig. 1). Together, these results suggest that a major gene controls two important characters related to grain size with a high heritability within this collection. 
Table 1

Descriptive statistics, broad sense heritability $\left(\mathrm{h}^{2}\right)$ and F-value of variance analysis for four agronomic traits in a collection of 159 wheat lines.

\begin{tabular}{|c|c|c|c|c|c|c|c|c|}
\hline \multicolumn{6}{|c|}{ Range } & \multicolumn{3}{|c|}{ F-values } \\
\hline Traits & Unit & Min & Max & Mean \pm SD & $h^{2}$ & Genotype (G) & Environment (E) & GxE \\
\hline Gle & $\mathrm{mm}$ & 1.22 & 8.55 & $3.2 \pm 0.08$ & 90.6 & $10.7^{\star \star \star \star}$ & 36.9 & 1.1 \\
\hline Gwi & $\mathrm{mm}$ & 0.45 & 3.45 & $1.6 \pm 0.04$ & 97.9 & $48.6^{\star \star \star}$ & 11.5 & 1.3 \\
\hline Gwe & $\mathrm{g}$ & 6.25 & 117.38 & $25.7 \pm 0.80$ & 61.6 & $30.9 * * *$ & $15.7^{\star \star \star}$ & $2.6^{*}$ \\
\hline Gyi & t/ha & 0.42 & 7.83 & $2.6 \pm 0.11$ & 56.0 & $66.3^{\star \star \star}$ & 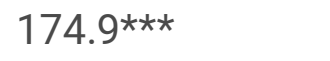 & $2.2^{\star}$ \\
\hline
\end{tabular}

Genome-wide SNP marker discovery and validation. To genetically characterize our wheat collection and study the genetic determinants of grain size, we used a double digestion (Pstl/Mspl) GBS approach to genotype this collection. Overall, 77,124 and 73,784 SNPs were discovered for the set of 71 Canadian wheat accessions and 159 exotics wheat accessions, respectively.

To assess the reproducibility and accuracy of genotypes called via the GBS approach, we genotyped 12 different plants of CS (i.e. biological replicates), which were added to the set of 288 wheat samples for SNP calling and bioinformatics analysis. Sequence reads of the full set of 300 wheat samples obtained from GBS were analyzed following the standard steps of SNP calling and bioinformatics analysis described below. This yielded a total of 129,940 loci that were used for the assessment of accuracy and reproducibility of SNP calls. For each individual plant of CS, the GBS calls were compared between replicates and with the Chinese Spring reference genome (at the corresponding positions).

On the non-imputed data, we detected a very high level of concordance (99.9\%) between the genotypes of each CS individual and the reference alleles for the 1,196,184 called genotypes ([130K SNPs x 12 samples] - missing data; Supplementary Fig. S1). Among those 12 biological replicates of CS, we found a very high reproducibility of genotype calls, as the pairwise identity of genetic distance calls varied from $1.56 \mathrm{E}-04$ to $5.08 \mathrm{E}-04$, with an average of $2.86 \mathrm{E}-04$. In order to ensure about identity of each CS plant, we have found that this value between the individual w56_Guelph (Canadian wheat variety) and each of the CS plant is greater than 0.1 .

After imputation of the missing genotype calls, we observed a mean concordance of $93.8 \%$ between the CS individuals and the CS reference genome. Furthermore, $76.7 \%$ of genotypes were called initially and $23.3 \%$ of genotypes were imputed. It should be noted that the accuracy rate for imputing missing data is 73.4\%. More details of SNP data set are provided in supplementary Table S1. 
As a further examination of data quality, we compared the genotypes called using both GBS and a SNP array on a subset of 71 Canadian wheat accessions that had been previously genotyped using the $90 \mathrm{~K}$ SNP array. A total of 77,124 GBS-derived and 51,649 array-derived SNPs were discovered in these 71 accessions (Supplementary Table S2). Of these, only 135 SNP loci were common to both platforms and among these potential 9,585 datapoints ( 135 loci $x 77$ lines), only 8,647 genotypes could be compared because the remaining 938 genotypes were missing in the array-derived data. As shown in Fig. 2, a high level of concordance $(95.1 \%)$ was seen between genotypes called by both genotyping approaches. To better understand the origin of discordant genotypes (4.9\%), we inspected the set of 429 discordant SNP calls and observed that: 1) $3.5 \%$ of discordant calls corresponded to homozygous calls of the opposite allele by the two technologies; and 2) $1.4 \%$ of discordant calls were genotyped as heterozygous by GBS while they were scored as homozygous using the 90K SNP array. More details are provided in Supplementary Table S3. From these comparisons, we conclude that GBS is a highly reproducible and accurate approach for genotyping in wheat and can yield a greater number of informative markers than the $90 \mathrm{~K}$ array.

Genome coverage and population structure. For the full set of accessions, a total of 129,940 SNPs was distributed over the entire hexaploid wheat genome. The majority of SNPs were located in the B $(61,844)$ and $A(50,106)$ sub-genomes compared to the $D$ (only 17,990 SNPs) sub-genome (Table 2). Although the number of SNPs varied 2- to 3-fold from one chromosome to another within a sub-genome, a similar proportion of SNPs was observed for the same chromosome across sub-genomes. Typically, around half of the markers were contributed by the B sub-genome ( $47.59 \%$ ), $38.56 \%$ by the A sub-genome and only $13.84 \%$ by the $D$ sub-genome.

Table 2

Distribution of SNP markers across the A, B and D genomes

\begin{tabular}{|c|c|c|c|c|}
\hline \multirow[t]{2}{*}{ Chromosomes } & \multicolumn{3}{|c|}{ Wheat genome } & \multirow[t]{2}{*}{ Total } \\
\hline & $A(*)$ & $\mathrm{B}\left({ }^{*}\right)$ & $D(*)$ & \\
\hline 1 & $6099(0.36)$ & $8115(0.48)$ & $2607(0.15)$ & $16821(0.13)$ \\
\hline 2 & $8111(0.35)$ & $11167(0.48)$ & $3820(0.17)$ & $23098(0.18)$ \\
\hline 3 & 6683(0.33) & 10555(0.53) & $2759(0.14)$ & 19997(0.15) \\
\hline 4 & $6741(0.58)$ & 4007(0.34) & $913(0.08)$ & 11661(0.09) \\
\hline 5 & $6048(0.38)$ & $8015(0.51)$ & $1719(0.11)$ & $15782(0.12)$ \\
\hline 6 & $5995(0.33)$ & $10040(0.55)$ & 2191(0.12) & $18226(0.14)$ \\
\hline 7 & $10429(0.43)$ & $9945(0.41)$ & $3981(0.16)$ & $24355(0.19)$ \\
\hline Total & 50106 & 61844 & 17990 & 129940 \\
\hline
\end{tabular}


The analysis of population structure for the 159 accessions of the association panel showed that $\mathrm{K}=6$ best captured population structure within this set of accessions and these clusters largely reflected the country of origin (Fig. 3). The number of wheat accessions in each of the six subpopulations ranged from 6 to 44. The largest number of accessions was found in northwestern Baja California (Mexico) represented here by Mexico 1 (44) and the smallest was observed in East and Central Africa (6).

GWAS analysis for marker-trait associations for grain size. To identify genomic loci contributing to grain size in wheat, we performed a GWAS analysis (159 accessions, 73,784 SNPs, grain length and width) using an CMLM approach.

As seen in Fig. 3, both Q-Q plots suggest that the confounding effects of population structure and relatedness were well controlled. For both traits, the greatest marker-trait associations were detected at the end of chromosome 2D, while another weaker association was shared at the beginning of chromosome 1D. For grain width only, a marker-trait association was detected on chromosome 4A. In total, seven SNPs were found to be associated with one or both traits, with respectively one, five and one significant SNPs being located on chromosomes 1D, 2D and 4A. Except for two SNPs (chr2D:442798939 and chr4A:713365388), all other SNPs were significant for both grain length and grain width. The SNP at 4A:713365388 was significant only for grain width while the SNP at 2D:442798939 was significant only for grain length.

The most significant association was observed on chromosome 2D and contributed to both grain length and grain width (Table 3; Fig. 3). For this QTL, a total of four SNPs was observed and the SNP most significantly associated to both traits was located at position 2D:452812899. A fifth SNP located at 2D:442798939 was significantly associated to grain length only, but was just below the significance threshold $(p$-value $=5.09 \mathrm{E}-05)$ for grain width . 
Table 3

Details of loci associated with grain size traits identified via a genome-wide association study in a collection of 159 hexaploid wheat lines.

\begin{tabular}{|c|c|c|c|c|c|c|c|}
\hline Loci & Chr & Grain traits & $P$-value & MAF & $R^{2}$ & Allelic effect & $\begin{array}{l}\text { Alleles } \\
\text { (Maj/Min) }\end{array}$ \\
\hline \multirow[t]{2}{*}{ chr1D:166874041 } & $1 \mathrm{D}$ & Length & 3.34E-06 & 0.29 & 0.06 & 0.25 & \multirow[t]{2}{*}{$\mathrm{T} / \mathrm{C}$} \\
\hline & & Width & 3.29E-05 & 0.29 & 0.06 & 0.11 & \\
\hline \multirow[t]{2}{*}{ chr2D:403935865 } & $2 \mathrm{D}$ & Length & $1.31 \mathrm{E}-06$ & 0.29 & 0.12 & 0.27 & \multirow[t]{2}{*}{$\mathrm{T} / \mathrm{C}$} \\
\hline & & Width & $1.29 \mathrm{E}-05$ & 0.29 & 0.07 & 0.12 & \\
\hline chr2D:442798939 & $2 \mathrm{D}$ & Length & 3.25E-06 & 0.28 & 0.11 & -0.26 & $A / G$ \\
\hline \multirow[t]{2}{*}{ chr2D:444560418 } & $2 \mathrm{D}$ & Length & 2.08E-06 & 0.28 & 0.11 & -0.27 & \multirow[t]{2}{*}{$A / G$} \\
\hline & & Width & 3.44E-05 & 0.28 & 0.06 & -0.12 & \\
\hline \multirow[t]{2}{*}{ chr2D:452644656 } & $2 \mathrm{D}$ & Length & $2.08 \mathrm{E}-06$ & 0.28 & 0.11 & -0.27 & \multirow[t]{2}{*}{$A / G$} \\
\hline & & Width & $3.44 \mathrm{E}-05$ & 0.28 & 0.06 & -0.12 & \\
\hline \multirow[t]{2}{*}{ chr2D:452812899 } & $2 \mathrm{D}$ & Length & $6.42 \mathrm{E}-07$ & 0.30 & 0.13 & -0.27 & \multirow[t]{2}{*}{$A / G$} \\
\hline & & Width & 7.03E-06 & 0.30 & 0.07 & -0.12 & \\
\hline chr4A:713365388 & $4 \mathrm{~A}$ & Width & $1.35 \mathrm{E}-05$ & 0.14 & 0.07 & 0.13 & $A / G$ \\
\hline
\end{tabular}

A high degree of LD was detected among some of the seven SNPs from chromosome 2D displaying association with grain traits. These formed one discontinuous linkage block as the LD between markers belonging to this block was higher (mean of $r^{2}=0.90$ ). For this reason, we considered these to define one quantitative trait locus (QTL) on chromosome 2D (Supplementary Fig. S2). This QTL included 5 SNP markers (chr2D:403935865, chr2D:442798939, chr2D:444560418, chr2D:452644656 and chr2D:452812899) and the peak SNP (chr2D:452812899) explained between 7\% and 13\% of the phenotypic variation for grain length and width. The minor allele frequencies (MAF) at this locus was 0.30 and exerted an allelic effect from -0.27 to $-0.12 \mathrm{~mm}$ (Table 3 ).

On chromosome 1D, the SNP marker chr1D:166874041 defined a QTL for both grain length and width. The percentage of phenotypic variation explained by this marker for grain length and width was $6 \%$ each, with a MAF of 0.29 and allelic effects of 0.25 and $0.11 \mathrm{~mm}$ for grain length and width, respectively. Furthermore, a high degree of interchromosomal LD was observed among the peaks SNPs between chromosomes $1 \mathrm{D}$ and $2 \mathrm{D}\left(\mathrm{r}^{2}=0.94\right)$ displaying association with grain traits. In addition, almost all 
accessions which have the major allele on chromosome 1D are the same which have the major allele on chromosome 2D. Thus, the combined impact of these two loci could explain the observed bimodal distribution.

On chromosome 4A, the SNP marker chr4A:713365388 defined a QTL for grain width only and it explained $7 \%$ of the variation, had a MAF of 0.14 and exerted an allelic effect of $0.13 \mathrm{~mm}$. However, we reported a very weak LD between this peak SNP marker and the two others on chromosomes 1D and 2D.

In summary, a total of three QTLs significantly associated with grain length and/or width were identified on chromosomes 1D, 2D and 4A.

Candidate gene detection for grain size. To identify candidate genes contributing to grain size within the studied wheat collection, we investigated the genes residing in the same linkage block as the peak SNP for each QTL. On chromosome 2D, the QTL with the largest number of associated SNPs (chr2D:403935865 to chr2D:452811303) included a total of 315 high-confidence genes. On chromosomes 1D and 4A, the SNP markers chr1D:166874041 and chr4A:713365388, defining each a QTL, respectively, doesn't included high-confidence genes. Upon examination of the annotations for these genes, the most promising candidate appears to be the TraesCS2D01G331100 gene in the QTL on chromosome 2D, an ortholog of the rice CYP724B1 gene, commonly known as the D11 gene. The D11 gene was previously reported as being involved in the regulation of internode elongation and seed development due to its role in the synthesis of brassinosteroids, key regulators of plant growth promoting the expansion and elongation of cells. More details are provided in Supplementary Table S4.

Haplotypes at the wheat orthologue of the rice D11 gene and their phenotypic effects. To provide a useful breeding tool for the main QTL identified in this research, we defined SNP haplotypes around our candidate gene. Using HaplotypeMiner, we identified two SNPs (chr2D:423365752 and chr2D:425474599, Supplementary Fig. S3) that best captured the SNP landscape in the vicinity of the candidate gene. These markers reside in the same haplotype block as the SNP markers, but were not individually found to be significantly associated with grain width and length. These SNP markers define three haplotypes (AT, CT or CC) around the candidate gene, with 100, 18 and 41 individuals carrying these haplotypes, respectively. To investigate the phenotypes associated with these haplotypes, we analyzed the trait value for each haplotype. Interestingly, we observed that for all traits, the mean values of accessions with haplotype AT were significantly larger $(p<0.001)$ than those obtained for the other haplotypes. As shown in Fig. 4, accessions carrying haplotype AT showed mean values of $3.76 \mathrm{~mm}$ for grain length, $2.03 \mathrm{~mm}$ for grain width, $41.64 \mathrm{~g}$ for grain weight and $2.6 \mathrm{t} / \mathrm{ha}$ for grain yield, compare to $2.43 \mathrm{~mm}, 1.12 \mathrm{~mm}, 26,57 \mathrm{~g}$ and $1.73 \mathrm{t} / \mathrm{ha}$ (respectively for grain length, width, weight and yield) for accessions carrying haplotype CC and $1.65 \mathrm{~mm}, 0.78 \mathrm{~mm}, 26.89 \mathrm{~g}$ and $1.69 \mathrm{t} / \mathrm{ha}$ (respectively for grain length, width, weight and yield) for accessions carrying haplotype CT. Furthermore, the relation between the 3 haplotypes and the 6 groups found in the population analysis showed that the haplotype AT predominates in the populations of Mexico 1 and North Africa (Supplementary Fig. S4, Supplementary Table S5). To conclude, we suggest that SNP markers corresponding to haplotype AT will provide a useful tool in marker-assisted breeding 
programs to improve wheat productivity. Therefore, we point out that the relationship between yield and haplotypes around the D11 gene would allow the selection of high-yielding wheat lines in a breeding program.

\section{Discussion}

The goal of our study was to identify genomic regions controlling variation for grain size in an international collection of 159 hexaploid wheat accessions through a GWAS approach. Thus, we collected the phenotypes for three grain traits (length, width, weight) in addition to grain yield. A statistical analysis revealed that the genotype was a major source of variance for all traits and that these exhibited a high heritability. In agreement with Arora et al. ${ }^{18}$ in Ae. tauschii and Rasheed et al. ${ }^{19}$ in wheat, we observed that grain length, grain width and grain weight were positively correlated to grain yield. Interestingly, a bimodal distribution was observed for both the grain length and width phenotypes, suggesting that one to a few major genes control these traits in our collection.

To assess the reproducibility and accuracy of genotypes called via the GBS approach, we genotyped 12 different plants of Chinese Spring (i.e. biological replicates), which were added to the set of 288 wheat samples for SNP calling and bioinformatics analysis, which yielded a total of 129,940 loci. Among the 12 biological replicates of CS, we found a very high reproducibility $(\sim 100 \%)$ in our genotype calls. Firstly, we verified the quality of our SNP data by investigating the reproducibility and accuracy of GBS-derived SNPs calls, and found that GBS-derived genotypes were in agreement with the reference genome in $99.9 \%$ of cases in over $1 \mathrm{M}$ comparisons for non-imputed data and $93.8 \%$ after imputation of the missing genotype calls. Recently, Abed et Belzile 20 reported that the accuracy of SNP calls was $99 \%$ for nonimputed and $89 \%$ for imputed SNPs dataset in Barley. In our study, $76.7 \%$ of genotypes were called initially, and only $23.3 \%$ were imputed. Thus, we conclude that the imputed data are of lower reliability.

As a further examination of data quality, we compared the genotypes called by GBS and a 90K SNP array on a subset of 71 Canadian wheat accessions. Among the 9,585 calls available for comparison, $95.1 \%$ of calls were in agreement. It is likely that both genotyping methods contributed to cases of discordance. It is known, however, that the calling of SNPs using the 90K array is challenging because of the presence of three genomes in wheat and the fact that most SNPs on this array are located in genic regions that tend to be typically more highly conserved, thus allowing for hybridization of homoeologous sequences to the same element on the array ${ }^{21,22}$. The fact that the vast majority of GBS-derived SNPs are located in noncoding regions makes it easier to distinguish between homoeologues ${ }^{21}$. This likely contributed to the very high accuracy of GBS-derived calls described above. We conclude that GBS can yield genotypic data that are at least as good as those derived from the 90K SNP array. This is consistent with the findings of Elbasyoni et al. ${ }^{23}$ as these authors concluded that "GBS-scored SNPs are comparable to or better than array-scored SNPs" in wheat genotyping. Likewise, Chu et al. ${ }^{24}$ observed an ascertainment bias for wheat caused by array-based SNP markers, which was not the case with GBS. 
Confident that the GBS-derived SNPs provided high-quality genotypic information, we performed a GWAS to identify which genomic regions control grain size traits. A total of three QTLs located on chromosomes 1D, 2D and 4A were discovered. Under these QTLs, seven SNPs were found to be significantly associated with grain length and/or grain width. Five SNPs were associated to both traits and two SNPs were associated to one of these traits. The QTL located on chromosome 2D shows a maximum association with both traits. Interestingly, previous studies have reported that the sub-genome D, originating from Ae. tauschii, was the main source of genetic variability for grain size traits in hexaploid wheat ${ }^{11,12}$. This is also consistent with the findings of Yan et al. ${ }^{15}$ who performed QTL mapping in a biparental population and identified a major QTL for grain length that overlaps with the one reported here. In a recent GWAS on a collection of Ae. tauschii accessions, Arora et al. ${ }^{18}$ reported a QTL on chromosome 2DS for grain length and width, but it was located in a different chromosomal region than the one we report here. With a view to develop useful breeding markers to improve grain yield in wheat, SNP markers associated to QTL located on chromosome 2D appear as the most promising.

To identify a candidate gene contributing to grain length and width, we examined the genes residing in the same linkage block as the peak SNP for each QTL. In the genomic interval spanned by the QTL contributing the most to the phenotypic variation for grain size (2D_40.4-45.1 Mb), a total of 315 highconfidence genes were observed. The TraesCS2D01G331100 gene seems like a highly promising candidate as it is orthologous to the rice CYP724B1 gene, commonly known as the D11 gene. The latter has been reported as involved in the regulation of internode elongation and seed development due to his role in brassinosteroid synthesis ${ }^{25}$. Brassinosteroids are a group of plant hormones and are key regulators of plant growth and development (including seeds) that promote cell expansion and elongation ${ }^{26}$.

To further refine the association between the TraesCS2D01G331100 gene and grain width and length, we defined SNP haplotypes. An analysis of haplotypes surrounding this gene identified three distinct haplotypes, and we observed that, for all grain size traits, the phenotypes corresponding to haplotype AT displayed significantly higher values than those of other haplotypes. We therefore suggest that SNP markers flanking TraesCS2D01G331100 could provide a useful tool in marker-assisted breeding programs to improve wheat productivity by selecting alleles leading to larger grain size and higher yield. In the longer term, it would be interesting to define more precisely the exact nature of the alleles at this gene through targeted re-sequencing of this gene in a broader collection of accessions.

\section{Materials And Methods}

Plant materials and phenotyping. A total of 230 hexaploid wheat (Triticum aestivum L.) varieties were used in our study. These accessions comprised two groups. A first group of 71 Canadian accessions was used to validate the accuracy of GBS in wheat. The second group of 159 accessions was used for genome-wide association analyses. Indeed, accessions were collected from many wheat breeding programs. Canadian accessions were collected from the University of Guelph Wheat Breeding Program 
and accessions from the second group were collected from South Africa through the Agricultural Research Council (ARC), Stellenbosch University's Plant Breeding Laboratory (SU-PBL) and SENSAKO's breeding programs, East Africa and Mexico via the International Maize and Wheat Improvement Center (CIMMYT), Central Africa by the Institute of Agricultural Research for Development (IRAD) and from farmers ${ }^{27}$, and North Africa per the International Center for Agricultural Research in the Dry Areas (ICARDA). With the latter accessions, field trials were conducted in two different trial sites in the bimodal humid forest zone of Cameroon, during the 2015-2016 wheat-growing seasons in Mbankolo (1057 m above sea level) and during 2016-2017 in Nkolbisson (650 m a. s. I.). In Mbankolo, the average temperature is $18-20^{\circ} \mathrm{C}$, bimodal rainfall with an annual average of $1600 \mathrm{~mm}$. In Nkolbisson, the annual average temperature is $23.5^{\circ} \mathrm{C}$, the rainfall is bimodal with an annual average of $1560 \mathrm{~mm}$. At each trial site, an incomplete alpha-lattice design with two replications was used. Each accession was planted in five-row plots, in 3-m rows with $5 \mathrm{~cm}$ between plants and $25 \mathrm{~cm}$ between rows. Then, fields trials were managed in accordance with the technical recommendations and standard agricultural practices for wheat ${ }^{28}$. Grain length (Gle), grain width (Gwi), 1000-grain weight (Gwe) and grain yield (Gyi) were recorded for each accession. Gle and Gwi were measured by a digital Vernier caliper on 20 seeds per variety randomly picked from a pool of grains from each harvested area ${ }^{18}$.

Analysis of phenotypic data. The analysis of variance for each trait was performed using PROC MIXED in SAS 9.4. Each cultivar was considered as a fixed effect, whereas replications and environments were considered as random effects. Pearson correlation coefficients between pairs of phenotypic traits were computed using Pearson's correlation in SPSS 20.0. We estimated the broad-sense heritability $\left(\mathrm{h}^{2}\right)$ for each trait using the following formula:

$$
h^{2}=\frac{V_{G}}{V_{G}+V_{G E}+V_{e}}
$$

where $\mathrm{V}_{\mathrm{G}}$ : genetic variance; $\mathrm{V}_{\mathrm{GE}}$ : genetic $\mathrm{x}$ environment variance and $\mathrm{V}_{\mathrm{e}}$ : error variance

DNA isolation, GBS library construction and sequencing. Genomic DNA was extracted from dried young leaf tissue $(\sim 5 \mathrm{mg})$ for all accessions using a CTAB DNA isolation method ${ }^{29}$. Then, DNA was quantified using a Quant-i $T^{\mathrm{TM}}$ PicoGreen (ThermoFisher Scientific, Canada) and the concentrations were normalized to $20 \mathrm{ng} / \mu \mathrm{l}$ for library preparation. Our 230 DNA samples were part of a larger set of 288 wheat samples on which GBS analysis was performed simultaneously (Fig. 5). In brief, 96-plex Pstl-Mspl GBS libraries were constructed $20,30,31$ and each was sequenced on three PI chips on an Ion Proton sequencer at the Plate-forme d'Analyses Génomiques of the Institut de Biologie Intégrative et des Systèmes (Université Laval, Québec, Canada). To allow an assessment of the quality of GBS-derived SNP calls, 12 independent samples of Chinese Spring (CS) DNA (each from a different plant) were used to produce a single (12plex) Pstl/Mspl library that was sequenced on one PI chip. 
Single nucleotide polymorphism calling and bioinformatics analysis. DNA sequences of the full set $(n=$ 300 ) of wheat samples obtained from GBS were analyzed using the Fast-GBS pipeline ${ }^{32}$ to align reads on the wheat reference genome (Chinese Spring v1.0) and to call SNPs. Fast-GBS results were first filtered to (i) keep only SNPs having the label "PASS" and SNPs positioned on chromosomes (i.e. not on scaffolds), (ii) remove indels and multiallelic SNPs, (iii) convert all heterozygous calls with genotype quality (GQ) < 30 to missing data, (iv) keep only SNPs with a minor allele count (MAC) $\geq 4$, (v) remove accessions with more than $80 \%$ of missing data, (vi) exclude SNPs with more than $10 \%$ heterozygotes and (vii) exclude SNPs with missing data $(\mathrm{N})>80 \%$. Finally, missing data were imputed using BEAGLE $v 5^{33}$ with the parameters described in Torkamaneh and Belzile ${ }^{34}$. Imputed genotypes were also filtered to keep only SNPs with a minor allele count $(M A C) \geq 4$. After these standard filtration steps, three subsets of accessions were extracted from the full dataset for specific goals: 1) establish the accuracy and reproducibility of GBS-derived SNP calls on 12 replicates of $\mathrm{cv}$. Chinese Spring; 2) compare SNP genotypes obtained via GBS and the $90 \mathrm{~K}$ array on a subset of 71 Canadian accessions and 3) perform GWAS for grain size on a diversity panel of 159 accessions. Additional filtration steps were performed on these subsets before these analyses. The imputed genotypes of the subset of 71 wheat accessions were filtered to keep only SNPs with a minor allele count (MAC) $\geq 4$ and exclude SNPs with more than $10 \%$ heterozygotes, while those from the collection of 159 wheat accessions were filtered to keep only SNPs with a minor allele frequency (MAF) $\geq 0.05$ (Fig. 5).

Validation of SNP call accuracy. The SNP genotypes for 12 different cv. Chinese Spring plants were used to assess the accuracy and reproducibility of GBS-derived SNP calls. Before and after imputation of missing data, we measured both the degree of agreement in SNP calls between replicates and the agreement between the GBS-derived SNP calls and the Chinese Spring reference genome V1.0 using an in-house script. To compare the accuracy of GBS-based and array-based genotype calls, we used a set of 71 Canadian wheat accessions for which genotypic data for 51,649 SNPs had been obtained previously using the $90 \mathrm{~K}$ SNP Infinium iSelect array ${ }^{35}$. For the 135 SNPs called in common using both methods, genotype calls were compared using an in-house script.

Population structure and linkage disequilibrium analyses. An analysis of population structure was performed on the collection of 159 wheat accessions using fastSTRUCTURE version $1.0^{36}$ on SNP markers filtered at MAF $\geq 0.05$ as recommended by Sobota et al. ${ }^{37}$. Population structure was evaluated using the filtered set of SNP markers using a simple prior and 1,000 iterations for K ranging from 1 to 12 . The optimal range of $\mathrm{K}$ was determined based on model complexity using the marginal likelihood method using the fastSTRUCTURE script chooseK.py, as well as on visualization of the log marginal likelihood, and population visualization using Distruct version $1.1^{38}$.

Genome-wide linkage disequilibrium (LD) analysis was performed using PLINK version $1.9^{39}$, via the Gabriel method ${ }^{40}$. This method is based on a confidence interval and a normalized measure of $D^{\prime}$. The pattern and distribution of intrachromosomal LD were visualized with LD plots generated using 
Haploview version $4.2^{41}$ to investigate the average LD decay along chromosomes. The smoothed seconddegree LOESS curve was fitted to determine the critical $D^{\prime}$ and $r^{2}$ between loci.

Genome-wide association study for grain traits. GWAS for grain traits was performed on the subset of 159 wheat accessions via the Genomic Association and Prediction Integrated Tool (GAPIT) version $2^{42}$. This approach, based on associations between the estimated genotypic values (BLUEs) for each trait and individual SNP markers ${ }^{43}$ was conducted with a compressed mixed linear model ${ }^{44}$. A matrix of genomic relationships among individuals (Supplementary Fig. S5) was calculated using the Van Raden method ${ }^{42}$. The statistical model used was: $Y=X \beta+Z u+\varepsilon$, where $Y$ is the vector of phenotypes; $\beta$ is a vector of fixed effects, including single SNPs, population structure (Q), and the intercept; $u$ is a vector of random effects including additive genetic effects as matrix of relatedness between individuals (the kinship matrix), $u \sim \mathrm{N}\left(0, \mathbf{K} \sigma_{a}{ }^{2}\right)$, where $\sigma_{a}{ }^{2}$ is the unknown additive genetic variance and $K$ is the kinship matrix; $\mathbf{X}$ and $\mathbf{Z}$ are the design matrices of $\beta$ and $u$, respectively; and $\boldsymbol{\varepsilon}$ is the vector of residuals, $\boldsymbol{\varepsilon} \sim \mathrm{N}\left(0, I \sigma_{e}{ }^{2}\right)$, where $\sigma_{e}{ }^{2}$ is the unknown residual variance and $I$ is the identity matrix. Association analysis was performed while correcting for both population structure and relationships among individuals with a combination of either the $\mathrm{Q}+\mathrm{K}$ matrices; $\mathrm{K}$ matrix was computed using the Van Raden method ${ }^{42}$. The Pvalue threshold of significance of the genome-wide association was based on false discovery rate (FDRadjusted $p<0.05)$.

Identification of candidate genes for grain size. To identify candidate genes affecting grain size in wheat, we defined haplotype blocks containing the peak SNP. Each region was visually explored for its LD structure and for genes known to reside in such regions. Then, we cross-referenced them against genes reported as controlling grain size in rice and other cereals in previous association and linkage mapping studies $^{15,18,45}$. By using the genome browser available for the wheat reference genome v1.0 on the International Wheat Genome Sequencing Consortium (IWGSC) website (https://urgi.versailles.inra.fr/jbrowseiwgsc/gmod_jbrowse), we extracted FASTA sequences representing the putative genes. Furthermore, the selected genes were further evaluated for their likely function based on publicly available genomic annotation. The function of these genes was also inferred by a BLAST of their sequences to the UniProt reference protein database (http://www.uniprot.org/blast/).

Identification of haplotypes around a candidate gene. To better define the possible alleles in a strong candidate gene, we used HaplotypeMiner ${ }^{46}$ to identify SNPs flanking the TraesCS2D01G331100 gene. For each haplotype, we calculated the trait mean (grain length, width, weight and yield) for lines sharing the same haplotype using the $\mathrm{R}$ ggpubr program ${ }^{47}$.

\section{Declarations}


Experiments on wheat are carried in accordance with national, international guidelines

\section{Acknowledgements.}

This work was supported by the Institut de Biologie Intégrative et des Systèmes (Université Laval, Québec, Canada), the Agence Universitaire de la Francophonie (AUF) and the MIC-CERES project jointly supported by Agropolis Fondation (grant AF Project ID 1301-003) through the Investissements d'avenir program (with reference number ANR-10-LABX-0001-01) and Fondazione Cariplo (grant FC Project ID 2013-1888). Authors are grateful to the International Maize and Wheat Improvement Center (CIMMYT), the International Center for Agricultural Research in the Dry Areas (ICARDA) and the Plant Breeding Laboratory (Department of Genetics, Stellenbosch University) for their technical supports and wheat varieties collection. They would like to thank the late Prof. Alireza Navabi for his significant contribution.

\section{Author contributions}

HT, MJ, ELMN, PFD and FB conceived the project. HT, PT, ELMN, PFD and WT contributed to sample collection, implemented and monitored the phenotyping trials in fields. BB provided the GBS data. $H T, S B$, $A A, D T, E L M N, B B, M J$ and FB contributed to genetic analyses, critical revision of the article and all authors contributed to final approval of the version to be published.

\section{Competing interests}

The authors declare no competing interests.

\section{Additional Information}

Supplementary information accompanies this paper are available at .......

\section{References}

1. Breseghello, F. \& Sorrells, M. E. QTL analysis of kernel size and shape in two hexaploid wheat mapping populations. Field Crops Res. 101, 172-179 (2007).

2. Gegas, V. C. et al. A Genetic Framework for Grain Size and Shape Variation in Wheat. Plant Cell. 22, 1046-1056 (2010).

3. Okamoto, Y., Kajimura, T., Ikeda, T. M. \& Takumi, S. Evidence from principal component analysis for improvement of grain shape-and spikelet morphology-related traits after hexaploid wheat speciation. Genes Genet. Syst. 87, 299-310 (2012).

4. Kuchel, H., Williams, K. J., Langridge, P., Eagles, H. A. \& Jefferies, S. P. Genetic dissection of grain yield in bread wheat. I. QTL analysis. Theor. Appl. Genet. 115, 1029-1041 (2007).

5. Gan, Y. \& Stobbe, E. H. Seedling Vigor and Grain Yield of 'Roblin' Wheat Affected by Seed Size. Agron. J. 88, 456-460 (1996). 
6. Marshall, D., Ellison, F. \& Mares, D. Effects of grain shape and size on milling yields in wheat. I. Theoretical analysis based on simple geometric models. Aust. J. Agric. Res. 35, 619 (1984).

7. Simons, K. J. et al. Molecular Characterization of the Major Wheat Domestication Gene Q. Genetics. 172, 547-555 (2006).

8. Williams, K., Munkvold, J. \& Sorrells, M. Comparison of digital image analysis using elliptic Fourier descriptors and major dimensions to phenotype seed shape in hexaploid wheat (Triticum aestivum L.). Euphytica. 190, 99-116 (2013).

9. Sun, X., Marza, F., Ma, H., Carver, B. F. \& Bai, G. Mapping quantitative trait loci for quality factors in an inter-class cross of US and Chinese wheat. Theor. Appl. Genet. 120, 1041-1051 (2010).

10. Tsilo, T. J., Hareland, G. A., Simsek, S., Chao, S. \& Anderson, J. A. Genome mapping of kernel characteristics in hard red spring wheat breeding lines. Theor. Appl. Genet. 121, 717-730 (2010).

11. Reynolds, M., Dreccer, F. \& Trethowan, R. Drought-adaptive traits derived from wheat wild relatives and landraces. J. Exp. Bot. 58, 177-186 (2006).

12. Rana, R. M., Bilal, M., Rehman, S. U., Iqbal, F. \& Shah, M. K. N. Synthetic wheat; a new hope for the hungry world. Asian J Agric Bio. 1, 91-94 (2013).

13. Okamoto, Y., Nguyen, A. T., Yoshioka, M., lehisa, J. C. M. \& Takumi, S. Identification of quantitative trait loci controlling grain size and shape in the $\mathrm{D}$ genome of synthetic hexaploid wheat lines. Breed. Sci. 63, 423-429 (2013).

14. Dvorak, J. et al. The Origin of Spelt and Free-Threshing Hexaploid Wheat. J. Hered. 103, 426-441 (2012).

15. Yan, L. et al. Identification of QTL for Grain Size and Shape on the D Genome of Natural and Synthetic Allohexaploid Wheats with Near-Identical AABB Genomes. Front. Plant Sci. 8, 1705 (2017).

16. Yu, J. \& Buckler, E. S. Genetic association mapping and genome organization of maize. Curr. Opin. Biotechnol. 17, 155-160 (2006).

17. Pang, Y. et al. High-Resolution Genome-wide Association Study Identifies Genomic Regions and Candidate Genes for Important Agronomic Traits in Wheat. Mol. Plant. 13, 1311-1327 (2020).

18. Arora, S. et al. Genome-Wide Association Study of Grain Architecture in Wild Wheat Aegilops tauschii. Front. Plant Sci. 8, 886 (2017).

19. Rasheed, A. et al. Genome-wide association for grain morphology in synthetic hexaploid wheats using digital imaging analysis. BMC Plant Biol. 14, 128 (2014).

20. Abed, A. et al. Genotyping-by-Sequencing on the lon Torrent Platform in Barley. in Barley (ed. Harwood, W. A.) vol. 1900 233-252 (Springer New York, 2019).

21. Deschamps, S., Llaca, V. \& May, G. D. Genotyping-by-Sequencing in Plants. Biology. 1, 460-483 (2012).

22. Torkamaneh, D., Boyle, B. \& Belzile, F. Efficient genome-wide genotyping strategies and data integration in crop plants. Theor. Appl. Genet. 131, 499-511 (2018). 
23. Elbasyoni, I. S. et al. A comparison between genotyping-by-sequencing and array-based scoring of SNPs for genomic prediction accuracy in winter wheat. Plant Sci. 270, 123-130 (2018).

24. Chu, J. et al. Suitability of Single-Nucleotide Polymorphism Arrays Versus Genotyping-By-Sequencing for Genebank Genomics in Wheat. Front. Plant Sci. 11, 42 (2020).

25. Tanabe, S. et al. A novel cytochrome P450 is implicated in brassinosteroid biosynthesis via the characterization of a rice dwarf mutant, dwarf11, with reduced seed length. Plant Cell. 17, 776-790 (2005).

26. Clouse, S. D. \& Sasse, J. M. Brassinosteroids: essential regulators of plant growth and development. Annu. Rev. Plant Biol. 49, 427-451 (1998).

27. Tékeu, H. et al. Genetic diversity of Cameroonian bread wheat (Triticum aestivum L.) cultivars revealed by microsatellite markers. Afr. J. Biotechnol. 16, 1832-1839 (2017).

28. Pask, A. J. D., Pietragalla, J., Mullan, D. M. \& Reynolds, M. P. Physiological breeding II: a field guide to wheat phenotyping(Cimmyt, 2012).

29. Doyle, J. J. \& Doyle, J. L. Isolation ofplant DNA from fresh tissue. Focus. 12, 39-40 (1990).

30. Elshire, R. J. et al. A Robust, Simple Genotyping-by-Sequencing (GBS) Approach for High Diversity Species. PLOS ONE. 6, e19379 (2011).

31. Poland, J. A., Brown, P. J., Sorrells, M. E. \& Jannink, J. L. Development of High-Density Genetic Maps for Barley and Wheat Using a Novel Two-Enzyme Genotyping-by-Sequencing Approach. PLoS ONE. 7, e32253 (2012).

32. Torkamaneh, D., Laroche, J., Bastien, M., Abed, A. \& Belzile, F. Fast-GBS: a new pipeline for the efficient and highly accurate calling of SNPs from genotyping-by-sequencing data. BMC Bioinformatics. 18, 1-7 (2017).

33. Browning, B. L., Zhou, Y. \& Browning, S. R. A One-Penny Imputed Genome from Next-Generation Reference Panels. Am. J. Hum. Genet. 103, 338-348 (2018).

34. Torkamaneh, D. \& Belzile, F. Scanning and Filling: Ultra-Dense SNP Genotyping Combining Genotyping-By-Sequencing, SNP Array and Whole-Genome Resequencing Data. PLOS ONE. 10, e0131533 (2015).

35. Wang, S. et al. Characterization of polyploid wheat genomic diversity using a high-density 90000 single nucleotide polymorphism array. Plant Biotechnol. J. 12, 787-796 (2014).

36. Raj, A., Stephens, M. \& Pritchard, J. K. fastSTRUCTURE: variational inference of population structure in large SNP data sets. Genetics. 197, 573-589 (2014).

37. Sobota, R. S. et al. Addressing population-specific multiple testing burdens in genetic association studies. Ann. Hum. Genet. 79, 136-147 (2015).

38. Rosenberg, N. A. DISTRUCT: a program for the graphical display of population structure. Mol. Ecol. Notes. 4, 137-138 (2004).

39. Purcell, S. et al. PLINK: A Tool Set for Whole-Genome Association and Population-Based Linkage Analyses. Am. J. Hum. Genet. 81, 559-575 (2007). 
40. Gabriel, S. B. et al. The structure of haplotype blocks in the human genome. Science. 296, 22252229 (2002).

41. Barrett, J. C., Fry, B., Maller, J. \& Daly, M. J. Haploview: analysis and visualization of LD and haplotype maps. Bioinformatics. 21, 263-265 (2005).

42. Lipka, A. E. et al. GAPIT: genome association and prediction integrated tool. Bioinforma. Oxf. Engl. 28, 2397-2399 (2012).

43. Abed, A., Belzile, F. \& Comparing Single -SNP, Multi-SNP, and Haplotype-Based Approaches in Association Studies for Major Traits in Barley. Plant Genome. 12, 190036 (2019).

44. Li, M. et al. Enrichment of statistical power for genome-wide association studies. BMC Biol. 12, 73 (2014).

45. Li, W. \& Yang, B. Translational genomics of grain size regulation in wheat. TAG Theor. Appl. Genet. Theor. Angew. Genet. 130, 1765-1771 (2017).

46. Tardivel, A., Torkamaneh, D., Lemay, M., Belzile, F. \& O’Donoughue, L. S. A Systematic Gene-Centric Approach to Define Haplotypes and Identify Alleles on the Basis of Dense Single Nucleotide Polymorphism Datasets. Plant Genome. 12, 180061 (2019).

47. Kassambara, A. ggpubr:"ggplot2" based publication ready plots (Version 0.1. 7). Obtido Desde HttpsCRAN R-Proj. Orgpackage Ggpubr (2018).

48. Torkamaneh, D. et al. Genome-wide association analyses reveal the genetic basis of biomass accumulation under symbiotic nitrogen fixation in African soybean. Theor. Appl. Genet. 133, 665$676(2020)$.

\section{Figures}



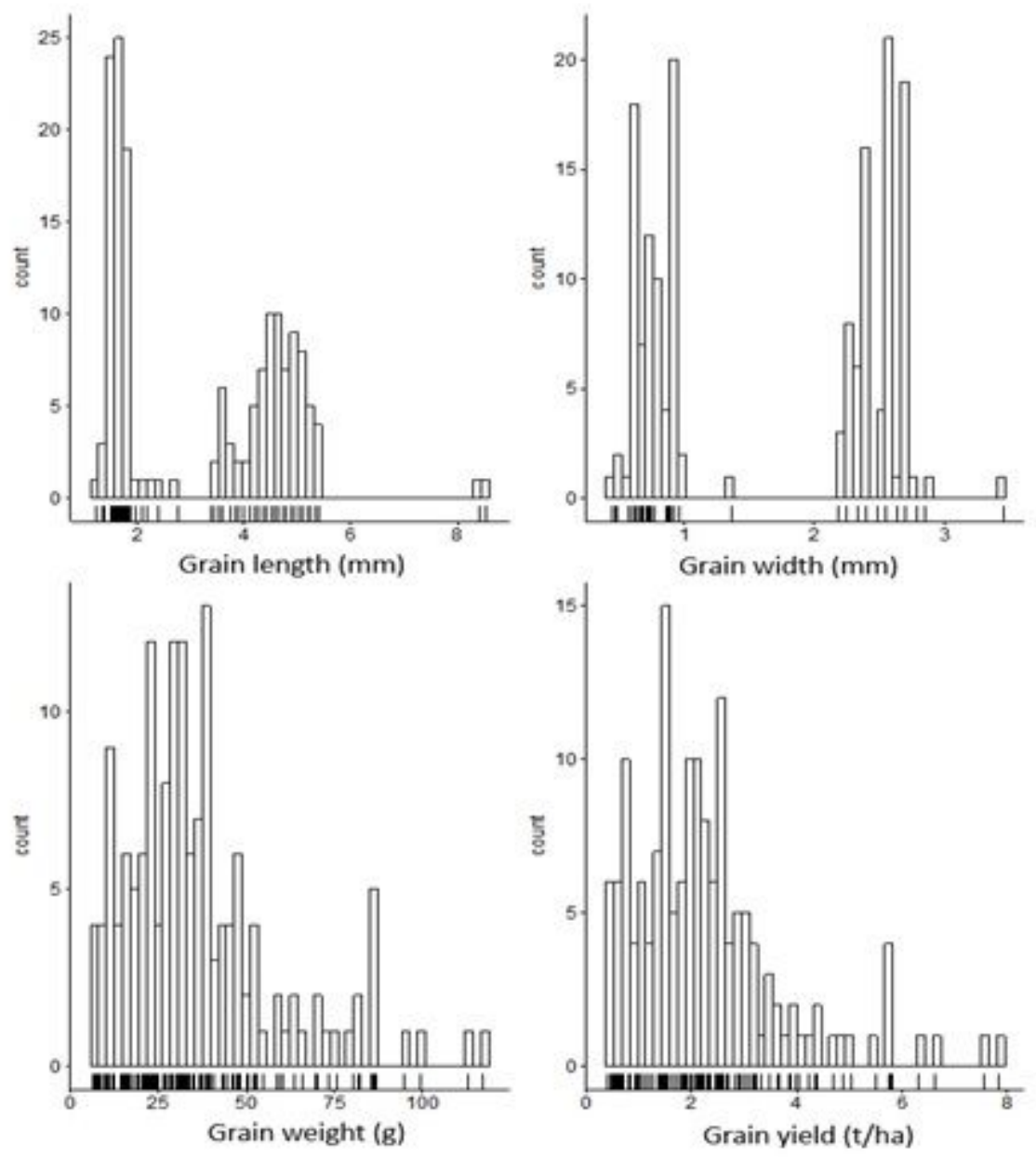

Figure 1

Distribution of phenotypes for grain length (upper left), grain width (upper right), grain weight (bottom left) and grain yield (bottom right). Histograms are based on the average trait value of each wheat line across the different environments. The bars under the histograms represent the density of individuals.

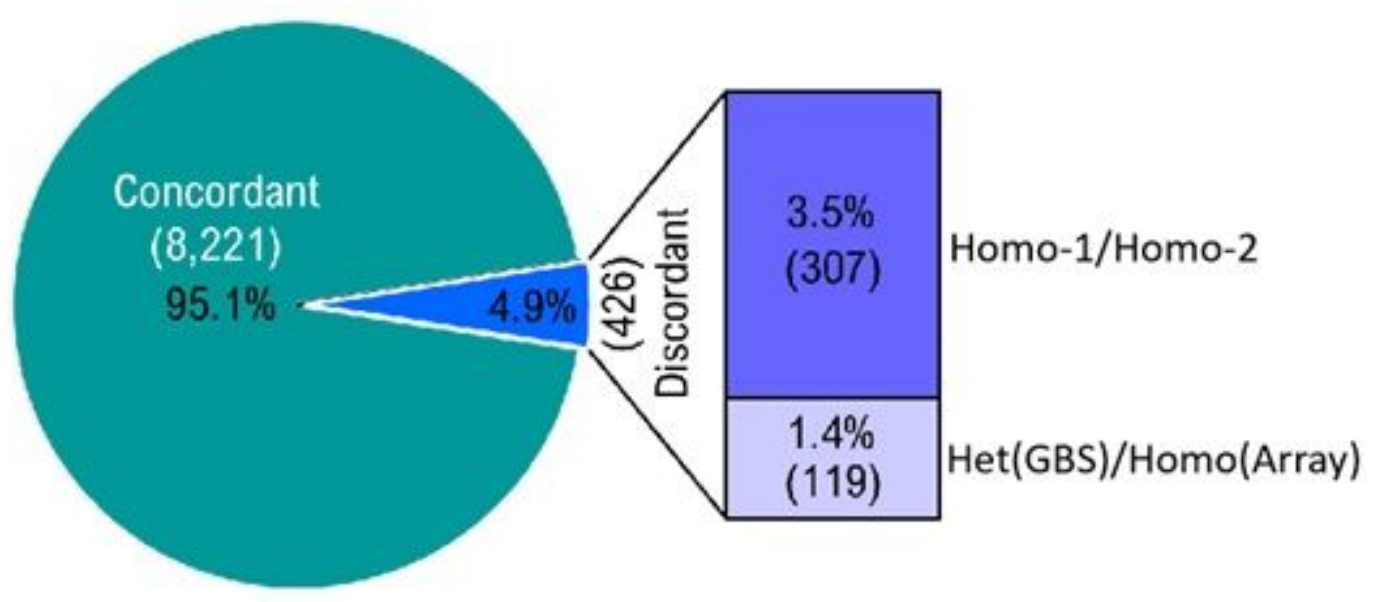


Figure 2

Concordance of genotype calls made using both marker platforms (GBS and 90K SNP Array). GBSderived SNP genotypes were compared to the genotypes called at loci in common with the 90K SNP Array for the same 71 wheat samples.

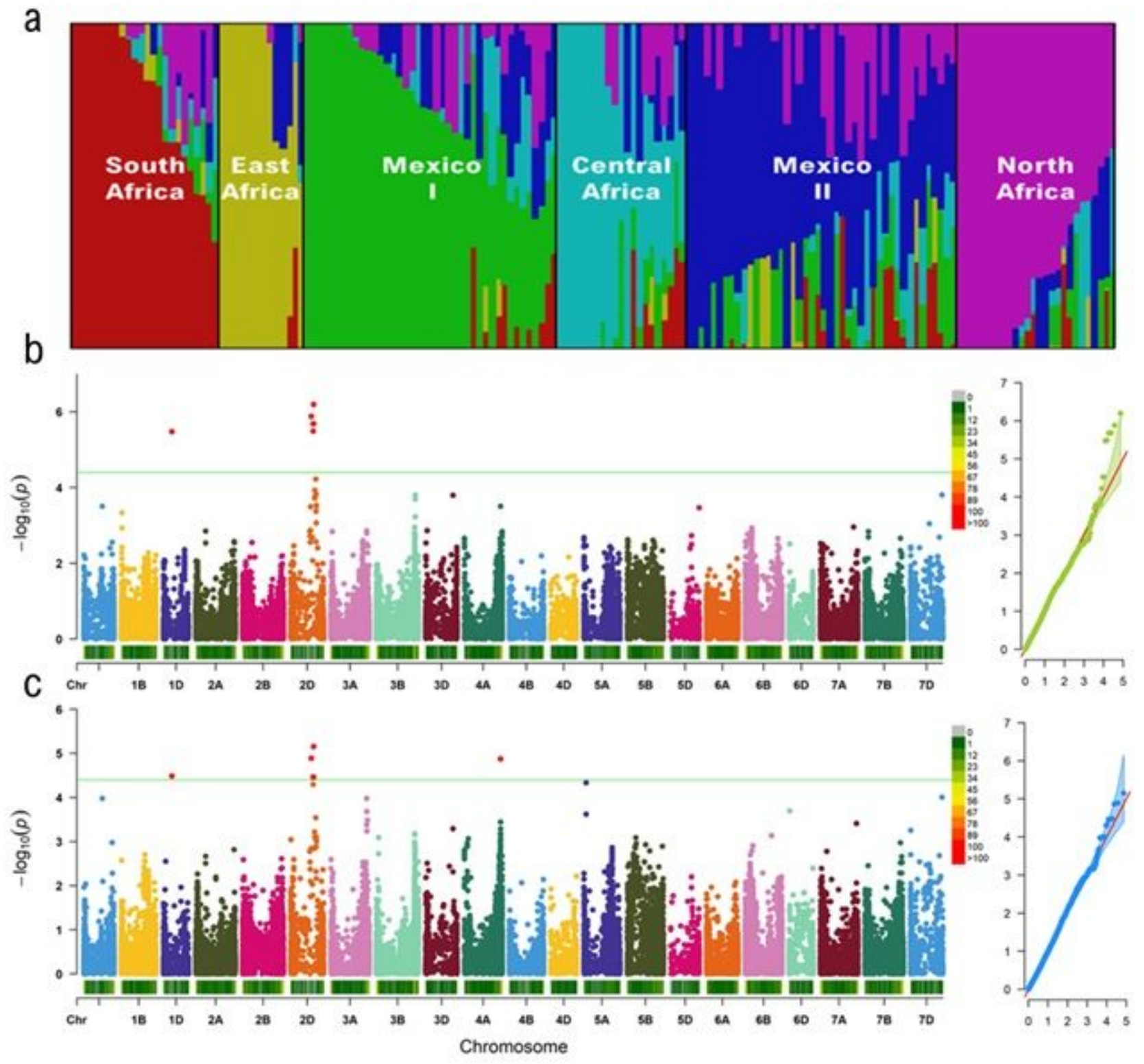

\section{Figure 3}

Population structure of 159 hexaploid wheat cultivars and genome-wide association studies of grain traits (a). Manhattan and Q-Q plots indicate the degree of association between SNPs and grain length (b) or grain width (c). 

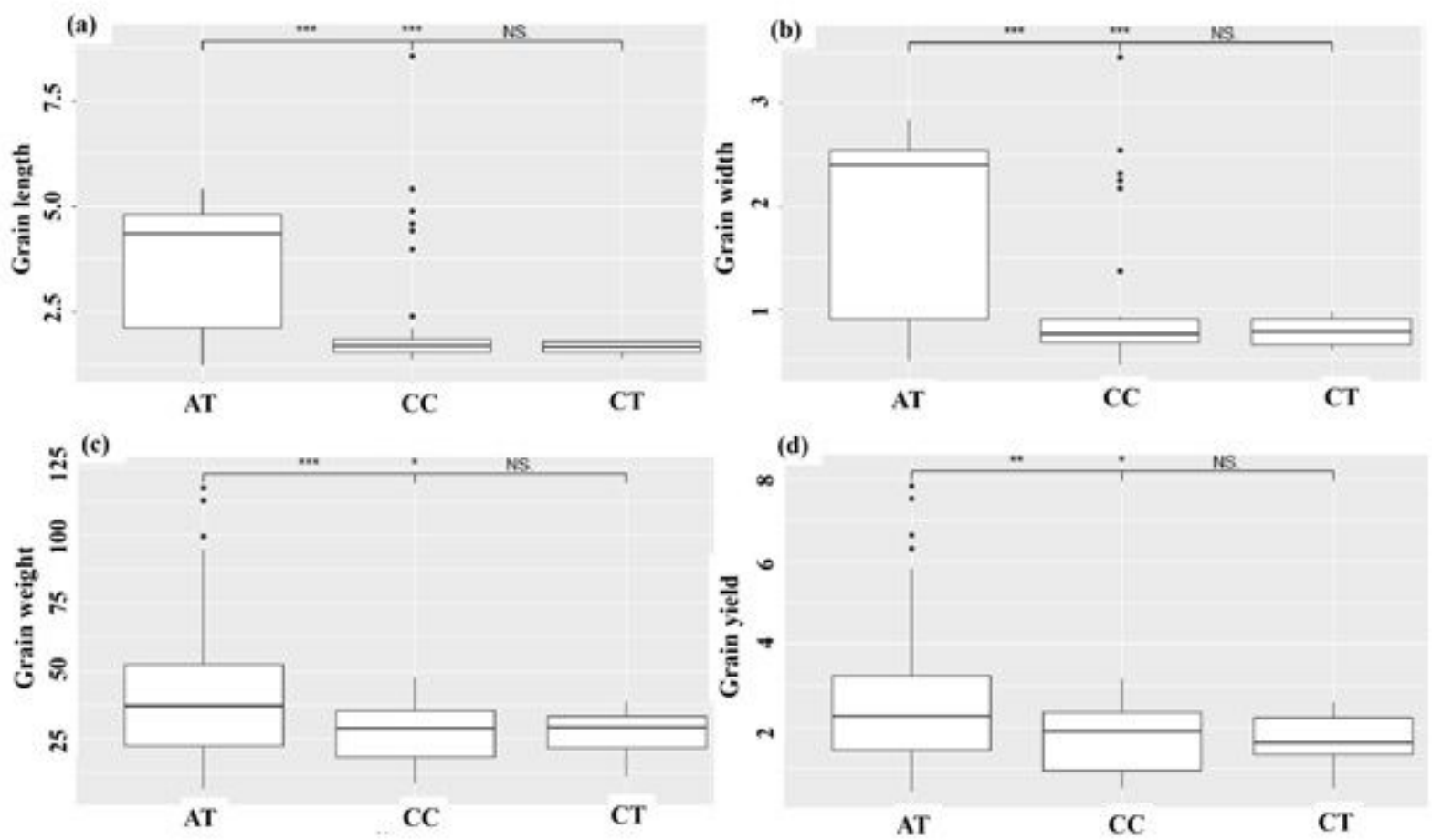

Figure 4

Impact of haplotypes on the grain traits and yield (using Wilcoxon test). Boxplots for the grain length (a), grain width (b), grain weight (c) and grain yield (d) are represented for each haplotype. ***: significant at $P<0.001$; **: significant at $P<0.01 ; *$ : significant at $P<0.1$; NS: non significant. 
Number and origin of accessions

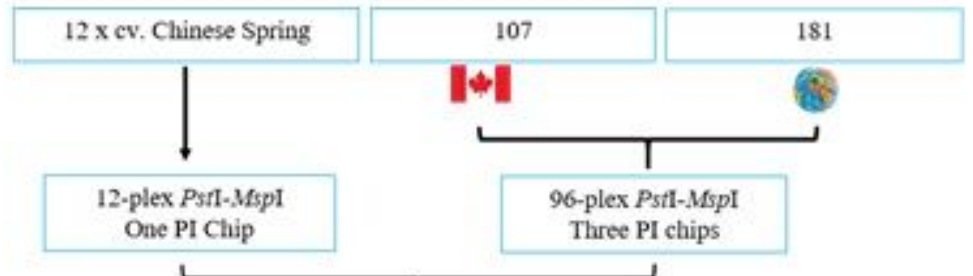

Multiplexing of libraries

Three PI chips

SNP calling and bioinformatics analysis

Extraction of subsets and additional filtration steps

Subsets of wheat accessions

Number of SNPS

Specifics goals
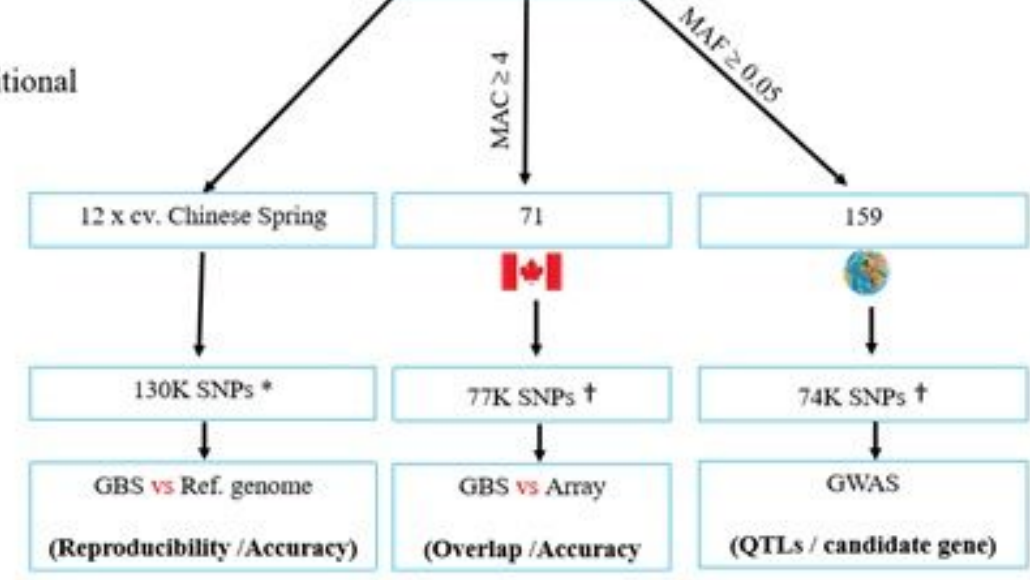

\section{Figure 5}

Schematic representation of the genetics analytical steps of wheat accessions subset. *vast majority of these are polymorphisms between Chinese Spring and the other accessions; $\uparrow$ these are SNPs that are polymorphic within the accessions of these sub-collections; MAC: Minor Allele Count; MAF: Minor Allele Frequency;

\section{Supplementary Files}

This is a list of supplementary files associated with this preprint. Click to download.

- SupplementaryFigure.docx

- SupplementaryTableS1..xlsx

- SupplementaryTableS2..xlsx

- SupplementaryTableS3..xlsx

- SupplementaryTableS4..xlsx

- SupplementaryTableS5..xlsx 